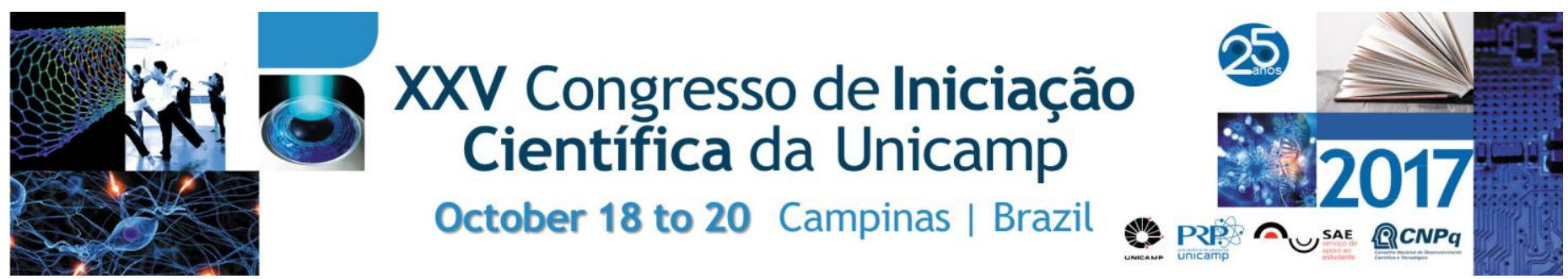

\title{
EFFECT OF AEROBIC EXERCISES ON COGNITIVE FUNCTION OF ELDERLY
}

\author{
Daisa Fabiele G. Moraes (IC), Amanda V. Sardeli (PG), Marina L. V. Ferreira (PG), Lucas C. Santos (IC), Claúdia \\ R. Cavaglieri (PQ), Mara Patrícia T. Chacon-Mikahil (PQ).
}

\begin{abstract}
Aging process leads to progressive loss of numerous physiological functions, including the cognitive functions (CF). Aerobic training $(\mathrm{AE})$ provides many beneficial adaptations to the health of elderly. More recently $\mathrm{AE}$ have shown improvements on CF. Furthermore, the improvements in CF can be seen also after an only session of AE. However, among many AE protocols, it is not clear, which of them could lead to higher CF improvements after only one session. We compared the effects of high load $A E$, low load $A E$ and low-load $A E$ with blood flow restriction. All of them were performed for $20 \mathrm{~min}$ in a treadmill by healthy elderly in a cross-over design. CF was assessed by Stroop Test before and after each AE protocol. None of the AE protocols tested in the present study improved the elderly cognitive function. These results could be due to the extremely healthy sample or the large heterogeneity of response among them.
\end{abstract}

Key words: aerobic exercise, cognitive function, aging, elderly.

\section{Introduction}

Aging process leads to progressive loss of numerous physiological functions, including the cognitive functions (CF). Aerobic training (AE) provides many beneficial adaptations to the health of elderly such as cardiovascular improvements and aerobic fitness and more recently $A E$ have shown improvements on $C F$. $A$ new type of $A E$, the low load $A E$ with blood flow restriction (LL-BFR), can increase muscle mass in addition to the known benefits of the traditional protocols of $A E$. Furthermore, the improvements in CF can be seen also after an only session of AE. Hypothetically, this type of exercise could increase CF to a different extent than low load (LL) and high load AE (HL), as seen LL-BFR leads to different changes in cardiac autonomic modulation and cardiac output (physiological mechanisms that modulate CF). Thus, the aim of the present study was to test the different $A E$ protocols effect on CF of healthy elderly.

\section{Results and Discussion}

Twenty-one elderly (8 men and 13 women, 63,8 \pm 4,2 years old) performed the three different-intensity protocols in a cross-over design: $\mathrm{HL}: 80 \% \quad \mathrm{VO}_{2} \max , \mathrm{LL}: 40 \%$ $\mathrm{VO}_{2} \mathrm{max}$ and LL-BFR: $40 \% \mathrm{VO}_{2} \mathrm{max}$ with $50 \%$ BFR. The Stroop Tests were made before and $5 \mathrm{~min}$ after all exercise protocols, which were performed in a treadmill for $20 \mathrm{~min}$. The data were presented just in mean, because the standard deviation was nearly 5 times larger than the mean (Figure $1 \mathrm{~A}$ and $1 \mathrm{~B}$ ). The mixed models analyses showed no time*session interactions (Figure $1 \mathrm{~A}$ and 1B). Whereas, there was a large heterogeneity among the data, as we showed the individual results for Stroop step 3 (Figure 1C-1E). Although many studies have shown the improvements in CF with $A E$, it did not happen in any of our AE protocols. It could be due to our extremely healthy sample with optimum baseline CF, or maybe just because of heterogeneity, as seen many of them increased number of hits and reduced time to answer the Stroop Test after AE.

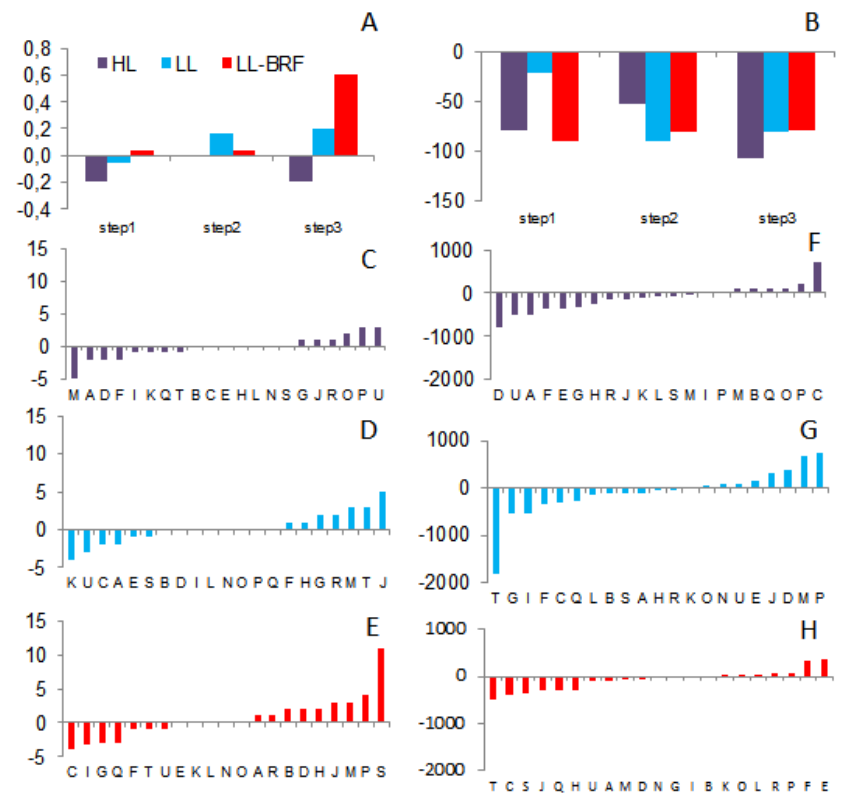

Figure 1. A: Mean of change in number of hits for all steps of Stroop following AE protocols. B: Mean of change in time to answer for all steps of Stroop following AE protocols. C-E: Individual changes in number of hits for Step 3. F-H: Individual changes in time to answer for Step 3.

\section{Conclusions}

We concluded the three types of aerobic exercise were not able to improve cognitive functions in this healthy elderly population.

\section{Acknowledgement}

We thank the support of PIBIC-CNPq, Laboratory of Exercise Physiology (FISEX), FEF, UNICAMP and LABFEF. 